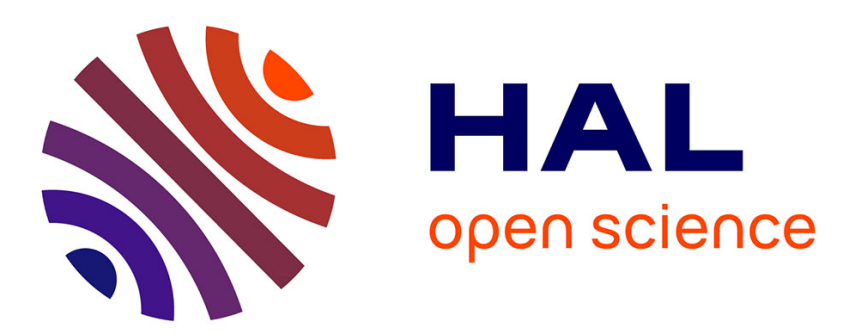

\title{
Simultaneous characterization of the electro-optic, converse-piezoelectric, and electroabsorptive effects in epitaxial "Sr,Ba...Nb2O6 thin films
}

Mireille Cuniot-Ponsard, Jean-Michel Desvignes, Alain Bellemain, Françoise Bridou

\section{To cite this version:}

Mireille Cuniot-Ponsard, Jean-Michel Desvignes, Alain Bellemain, Françoise Bridou. Simultaneous characterization of the electro-optic, converse-piezoelectric, and electroabsorptive effects in epitaxial "Sr,Ba...Nb2O6 thin films. Journal of Applied Physics, 2011, 109 (1), pp.014107. 10.1063/1.3514083 . hal-00555291

\section{HAL Id: hal-00555291 \\ https://hal-iogs.archives-ouvertes.fr/hal-00555291}

Submitted on 2 Apr 2012

HAL is a multi-disciplinary open access archive for the deposit and dissemination of scientific research documents, whether they are published or not. The documents may come from teaching and research institutions in France or abroad, or from public or private research centers.
L'archive ouverte pluridisciplinaire HAL, est destinée au dépôt et à la diffusion de documents scientifiques de niveau recherche, publiés ou non, émanant des établissements d'enseignement et de recherche français ou étrangers, des laboratoires publics ou privés. 


\section{AIP Appilied Physics}

\section{Simultaneous characterization of the electro-optic, converse-piezoelectric, and electroabsorptive effects in epitaxial $(\mathrm{Sr}, \mathrm{Ba}) \mathrm{Nb} 2 \mathrm{O} 6$ thin films}

M. Cuniot-Ponsard, J. M. Desvignes, A. Bellemain, and F. Bridou

Citation: J. Appl. Phys. 109, 014107 (2011); doi: 10.1063/1.3514083

View online: http://dx.doi.org/10.1063/1.3514083

View Table of Contents: http://jap.aip.org/resource/1/JAPIAU/v109/i1

Published by the American Institute of Physics.

\section{Related Articles}

Correlation between oxygen stoichiometry, structure, and opto-electrical properties in amorphous In2O3:H films J. Appl. Phys. 111, 063721 (2012)

Dynamic response of a polymer-stabilized blue-phase liquid crystal

J. Appl. Phys. 111, 063103 (2012)

Unusual broadening of $\mathrm{E} 0$ and $\mathrm{EO}+\Delta \mathrm{SO}$ transitions in GaAsBi studied by electromodulation spectroscopy J. Appl. Phys. 111, 066103 (2012)

Electric field effects on reduced effective masses of minibands at the mini-Brillouin-zone center and edge in a GaAs/AIAs superlattice

J. Appl. Phys. 111, 053523 (2012)

Spontaneous induction of the uniform lying helix alignment in bimesogenic liquid crystals for the flexoelectro-optic effect

Appl. Phys. Lett. 100, 063501 (2012)

\section{Additional information on J. Appl. Phys.}

Journal Homepage: http://jap.aip.org/

Journal Information: http://jap.aip.org/about/about_the_journal

Top downloads: http://jap.aip.org/features/most_downloaded

Information for Authors: http://jap.aip.org/authors

\section{ADVERTISEMENT}
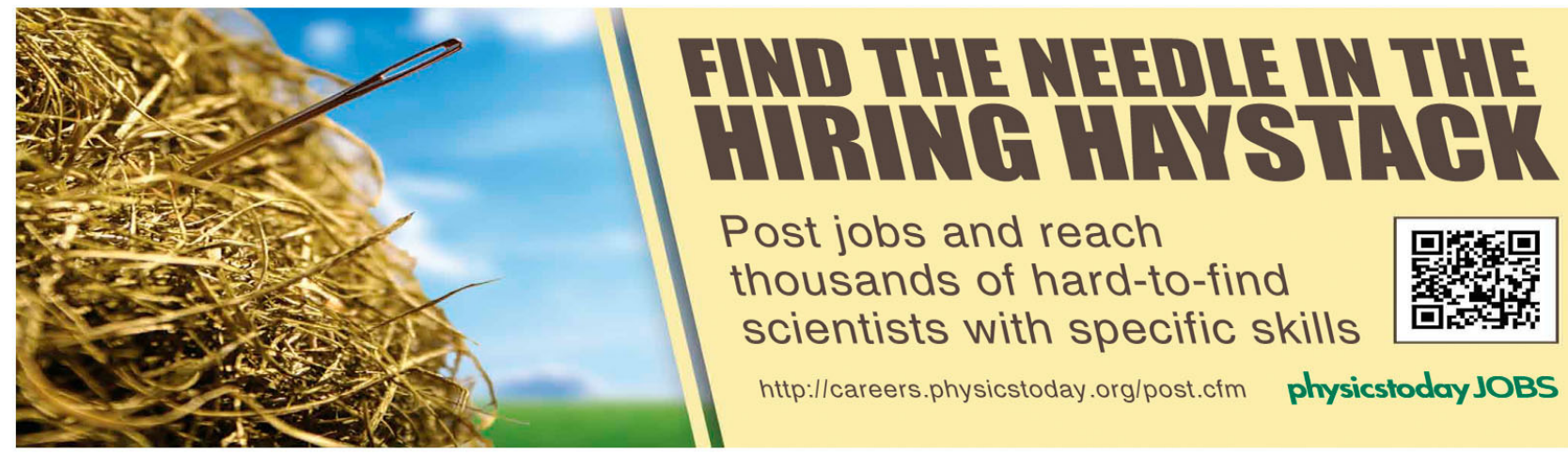


\title{
Simultaneous characterization of the electro-optic, converse-piezoelectric, and electroabsorptive effects in epitaxial $(\mathrm{Sr}, \mathrm{Ba}) \mathrm{Nb}_{2} \mathrm{O}_{6}$ thin films
}

\author{
M. Cuniot-Ponsard, ${ }^{\text {a) }}$ J. M. Desvignes, A. Bellemain, and F. Bridou \\ Laboratoire Charles Fabry de l'Institut d'Optique, CNRS, Univ Paris-Sud, 2 Avenue Augustin Fresnel, \\ RD 128, 91127 Palaiseau Cedex, France
}

(Received 21 July 2010; accepted 9 October 2010; published online 7 January 2011)

\begin{abstract}
Implementation of the linear electro-optic (EO) effect in thin film waveguides is expected to allow drastic reductions in the drive voltage, power, and dimensions of devices devoted to light modulation. It should also enable the realization of electrically tunable photonic crystal devices. In this paper we introduce a method which eliminates systematically the sources of the unreliability which strongly affects thin film EO characterization. Based on a Fabry-Perot reflective configuration, the method enables characterizing simultaneously the EO, converse-piezoelectric, and electroabsorptive effects in a film. It provides the magnitude and sign of each of the involved coefficients, and allows accounting for the whole of experimental data versus angle of incidence for both transverse-electric and transverse-magnetic polarizations. At $\lambda=633 \mathrm{~nm}$ and room temperature, the results obtained with an epitaxial strontium barium niobate $\left(\mathrm{Sr}_{\mathrm{x}} \mathrm{Ba}_{1-\mathrm{x}} \mathrm{Nb}_{2} \mathrm{O}_{6}, \mathrm{x}\right.$ $=0.60)$ ferroelectric thin film, are: $r_{13}=+8.5 \pm 1.3 \mathrm{pm} / \mathrm{V}, \quad r_{33}=+38.9 \pm 0.5 \mathrm{pm} / \mathrm{V}, d_{33}$ $=\Delta e / \Delta V=+21 \pm 4 \mathrm{pm} / \mathrm{V}$, and $\Delta k_{o} / \Delta V=(+9.8 \pm 0.6) \times 10^{-6}$, where $r_{13}$ and $r_{33}$ are two linear EO coefficients, $d_{33}$ is a converse-piezoelectric coefficient, and $e, k_{o}, V$ represent, respectively, the film thickness, film ordinary extinction coefficient, and applied voltage. Converse-piezoelectric and electroabsorptive effects are found significant in the film response at a frequency below piezoelectric resonance. Diagonal and effective EO coefficients of the $(\mathrm{Sr}, \mathrm{Ba}) \mathrm{Nb}_{2} \mathrm{O}_{6}(\mathrm{SBN})$ film explored in the present work are larger than those of a crystal of lithium niobate (LN) at the same wavelength $\lambda=633 \mathrm{~nm}$. Taking into account the significant difference in dielectric permittivity between the two materials, advances and potential of LN and SBN thin film paths are compared.

(C) 2011 American Institute of Physics. [doi:10.1063/1.3514083]
\end{abstract}

\section{INTRODUCTION}

Electro-optic (EO) light modulation is a key function in light-wave technologies. It is mostly realized by exploiting the linear EO Pockels effect in ferroelectric bulk crystals like lithium niobate (LN) for primary example. Pockels effect may be expressed in terms of an electric-field-induced distortion of the refractive index ellipsoid as

$$
\Delta\left(\frac{1}{n_{i j}^{2}}\right)=\sum_{k} r_{i j k} E_{k},
$$

where $n, r$, and $E$ represent, respectively, refractive index, EO coefficient, and electric-field. Optimizing the performance of an EO modulator involves minimizing the halfwave voltage-length product $\left(V_{\pi} L\right)$ and the drive power $(P)$. Given a propagation distance under electric-field $(L), V_{\pi}$ is the applied voltage that induces a $\pi$ phase shift in the optical wave emerging from the crystal, and $P$ is the drive power required. If we consider a plane wave propagating through a LN crystal, polarized linearly parallel to the extraordinary optical axis and to the applied electric-field (Fig. 1), the two quantities to be minimized may be expressed as ${ }^{1}$

$$
V_{\pi} L=\frac{\lambda d}{n_{e}^{3} r_{33}},
$$

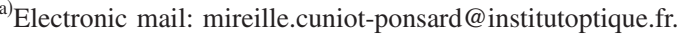

$$
P=C \lambda V_{\pi} w \frac{\varepsilon_{3} \Delta \nu}{n_{e}^{3} r_{33}},
$$

where $\lambda$ is the light wavelength, $d$ is the distance between electrodes, $n_{e}$ is the extraordinary refractive index, $r_{33}$ and $\varepsilon_{3}$ are, respectively, the linear EO coefficient and relative dielectric permittivity involved in this configuration (using reduced notation for $r_{333}$ and $\varepsilon_{33}$ ), $\mathrm{C}$ is a constant, $w$ is the width of electrodes, and $\Delta \nu$ is the frequency bandwidth of the modulator.

Expressions (2) and (3) indicate that an optimum geometry is achieved when minimizing the two transverse dimen-

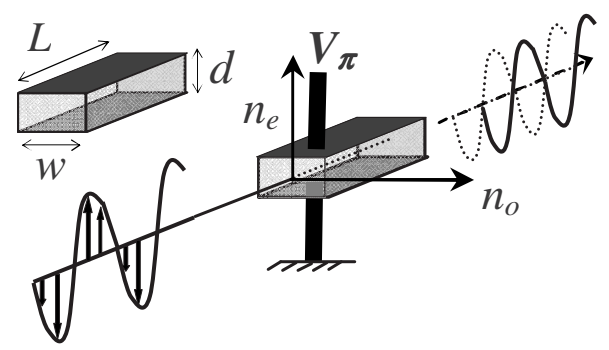

FIG. 1. Principle of EO light modulation based on Pockels linear EO effect. An uniaxial crystal, like LN or SBN, is chosen as an example. Applied voltage $V$ induces a variation in extraordinary refractive index $\Delta n_{e}$ $=\left(-n_{e}^{3} / 2\right) r_{33}(V / d)$ and a consequent phase shift $\Delta \varphi=(2 \pi / \lambda) L \Delta n_{e}$ of the propagating wave linearly polarized along the extraordinary axis. $r_{33}$ is the diagonal EO Pockels coefficient and $\lambda$ is the light wavelength. The halfwave voltage $V_{\pi}=\lambda d /\left(\operatorname{Lr}_{33} n_{e}^{3}\right)$. 
sions ( $d$ and $w$ ), provided the optical wave is successfully confined in the region under electric-field. A considerable decrease in the required $V_{\pi} L$ and $P$ values, by three orders of magnitude, is, therefore, expected from the replacement of bulk crystals by thin film waveguides about $1 \mu \mathrm{m}$ thick. A further reduction in the power consumption may be achieved by using a strip guide configuration where the optical wave is confined in both transverse dimensions. These geometric considerations have led to develop waveguides produced either by increasing refractive index in a shallow region below the surface of a bulk crystal, or prepared by epitaxial deposition, or by transfer, of a thin film onto a substrate of lower refractive index. Compared to the first ones, thin film techniques allow a better optical confinement, a better control of transverse dimensions, and a lower distance $d$ between electrodes. By using crystal ion slicing (CIS) and wafer bonding technique to prepare single-crystalline thin films, Rabiei and Günter ${ }^{2}$ decreased the distance between electrodes until a value of $1.8 \mu \mathrm{m}$ without degrading the EO coefficient of initial LN crystal $\left(r_{33}=31 \mathrm{pm} / \mathrm{V}\right)$, thus potentially reducing the $V_{\pi} L$ product of $\mathrm{LN}$ at $\lambda=633 \mathrm{~nm}$ and in the configuration of Fig. 1 to a value as low as $0.3 \mathrm{~V} \mathrm{~cm}$. With thin film epitaxial deposition the difficulty does not lie in the reduction in distance between electrodes but in the preparation of a thin film material with high EO properties. The highest value reported for $r_{33}$ at $\lambda=633 \mathrm{~nm}$ in a $\mathrm{LN}$ thin film prepared by deposition on a substrate is about $18 \mathrm{pm} / \mathrm{V},{ }^{3}$ which should allow a $V_{\pi} L$ value as low as that obtained with CIS technique, i.e., close to the theoretical crystalline lower limit for LN.

Any further significant improvement in EO modulation performance can then only arise from changing $\mathrm{LN}$ for a material with better EO properties, that is, values of $n, r$, and $\varepsilon$ which allow reducing $V_{\pi} L$ without increasing $P$. Beside $\mathrm{LN}$, the ferroelectric materials which have been considered in the literature in view of preparing $\mathrm{EO}$ thin films are mainly $\mathrm{BaTiO}_{3}{ }^{4,5}(\mathrm{Ba}, \mathrm{Sr}) \mathrm{TiO}_{3},{ }^{6,7}(\mathrm{~Pb}, \mathrm{La})(\mathrm{Zr}, \mathrm{Ti}) \mathrm{O}_{3},{ }^{8-10}$ and $(\mathrm{Sr}, \mathrm{Ba}) \mathrm{Nb}_{2} \mathrm{O}_{6}(\mathrm{SBN}) .{ }^{11-14}$ EO coefficients higher than that of LN crystal by more than one order of magnitude have been reported for some of these films. $5,9,12$

In addition to increasing drastically EO modulation performance, the implementation of Pockels EO effect in thin film waveguides opens up the path to the realization of electrically tunable photonic crystal (PC) devices. Through the engineering of photonic band gaps, PC structures enable developing the functionality and reducing radically the size of optical devices. Position and shape of a photonic band gap are dependent on refractive index of the propagating medium and therefore can be electro-optically controlled. This EO control considerably broadens the scope of PC structures potential functionality. For example, instead of being achieved by using either a Mach-Zehnder configuration or the relative retardation between orthogonal polarization components, light intensity switching can potentially be very compactly accomplished in a single channel waveguide by electrically inducing a shift in wavelength of the photonic bandgap. The potential of EO controlled PC structures has been investigated in the literature through simulations ${ }^{15,16}$ and experi- mental demonstrations. ${ }^{17-19}$ The future deployment of photonic technology largely rests on the tunability of PC characteristics.

The great potential of EO thin films is linked to our ability to prepare highly EO and well-controlled thin films. A reliable method of characterizing the film electric-fieldinduced response is an essential tool for progressing in this field. Film response is a combination of conversepiezoelectric (CPE), EO and electroabsorptive (EA) effects. In this paper, we present a method which allows determining simultaneously these three contributions. We have developed it with the aim of increasing the reliability of thin film EO coefficients determination, and its main specific feature is a required high level of self-consistency. Section II discusses the sources of errors in thin film EO characterization and details the principle of the method introduced in the present work. Section III deals with the preparation and properties of epitaxial strontium barium niobate $(\mathrm{SBN})$ thin films we have developed. Section IV presents the successive steps and results of the characterization procedure applied to one of these SBN thin films. Section V discusses the results with regard to those reported in the literature for SBN thin films or SBN crystals.

\section{CHARACTERIZATION OF A THIN FILM ELECTRIC- FIELD-INDUCED RESPONSE}

\section{A. Usual experimental approaches and difficulties}

Methods used to determine the EO coefficients of a thin film may be classified in three types: interferometric, polarimetric, and prism-coupling methods. In interferometric methods, ${ }^{12,20-23}$ an interference pattern is created with light beams, at least one of which propagates inside the EO film before being either transmitted or reflected. Such a pattern may be obtained by various ways like the use of a MachZehnder configuration, ${ }^{20}$ a Fabry-Perot configuration, ${ }^{21-23}$ or a diffraction grating. ${ }^{12}$ Electric-field-induced response of the active film causes a variation in phase and amplitude of the wave emerging from the film, and results in a variation $(\Delta I)$ in pattern light intensity. The different EO coefficients are determined independently from measurement of $\Delta I$, by varying the direction of the linear polarization. Polarimetric methods $^{24-26}$ exploit the anisotropy of EO response. Electricfield induces a change $(\Delta \varphi)$ in the relative phase between the two orthogonal components of a linearly polarized light beam. Light may be either transmitted ${ }^{24}$ or reflected ${ }^{25,26}$ by the film and in most cases $\Delta \varphi$ is converted into an output light intensity variation $\Delta I$ by using an analyzer. From measurement of $\Delta \varphi$ or $\Delta I$, an "effective" EO coefficient is determined, which is a linear combination of two EO coefficients. Polarimetric methods do not enable determining separately the value of each EO coefficient. In the prismcoupling technique ${ }^{27}$ (also called ATR for attenuated total reflection) the film is sandwiched between two electrodes and forms a waveguide in which light is coupled by using a prism. The electric-field-induced response of the film modifies coupling incident angles, thus causing a variation $\Delta I$ in the intensity of the light beam emerging from the prism. 


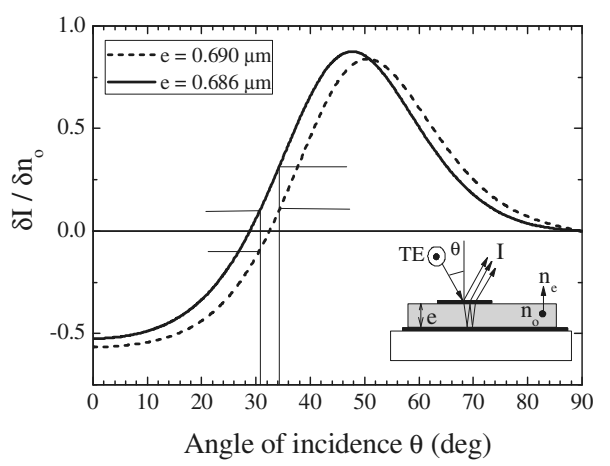

FIG. 2. Example of evolution of the derivative $\partial I / \partial n_{o}$ when slightly modifying one parameter, here, the EO film thickness value $e$, in a Fabry-Perot interferometric setup. Output reflected intensity $I$ and derivative $\partial I / \partial n_{o}\left(n_{o}\right.$ is the ordinary refractive index of active film) have been rigorously calculated vs incident angle for transverse electric polarization TE without using any simplifying approximation. A variation in film thickness of only $+0.6 \%$ $(e=0.686 \rightarrow 0.690 \mu \mathrm{m})$ can strongly modify the derivative value: $\partial I / \partial n_{o}$ is multiplied by $(-1)$ if measurement is performed at $\theta \approx 31^{\circ}$, and by $(1 / 3)$ if measurement is performed at $\theta \approx 34^{\circ}$.

In all cases, determination of EO coefficients is performed from a measurement of the variation $\Delta I$ induced in output light intensity. The electric-field-induced response being a priori a combination of $\mathrm{EO}$, converse piezoelectric and EA effects, the induced output signal $\Delta I$ is a function of the variations in refractive index $\left(\Delta n_{i j}\right)$, in dimensions $\left(\Delta e_{i}\right)$, and in extinction coefficients $\left(\Delta k_{i j}\right)$, which may be expressed as

$$
\Delta I=\sum_{i, j} \frac{\partial I}{\partial n_{i j}} \Delta n_{i j}+\sum_{i} \frac{\partial I}{\partial e_{i}} \Delta e_{i}+\sum_{i, j} \frac{\partial I}{\partial k_{i j}} \Delta k_{i j}
$$

Besides $\Delta I$ measurement uncertainty, two sources of potential error affect the determination of EO coefficients. The first is the use of approximations in view of simplifying the above expression (4): they consist in neglecting optical effects like multiple reflections, and/or some of the electricfield-induced effects like converse-piezoelectricity and electroabsorption. The group of Y. Levy demonstrated the significant errors that can be induced by such approximations in the two cases of a prism-coupling ${ }^{28}$ and of a polarimetric method. ${ }^{29}$ The second source of potential error is the extreme sensitivity of derivatives appearing in Eq. (4) to a variation in the values of physical parameters of the films interacting with light (active film and electrodes). An illustration of this sensitivity is given in Fig. 2. The example of a Fabry-Perot interferometric setup has been chosen. The output reflected intensity $I$ and the derivative $\partial I / \partial n_{o}\left(n_{o}\right.$ is the ordinary refractive index of the active film) have been rigorously calculated for transverse electric (TE) polarization without using any simplifying approximation. Figure 2 shows the plots of $\partial I / \partial n_{o}$ versus angle of incidence $\theta$ calculated from two sets of parameters with a small change only in the active film thickness value ( $e=0.686$ or $0.690 \mu \mathrm{m})$. The comparison between the two plots shows that if the actual thickness value is $0.686 \mu \mathrm{m}$ instead of a believed value of $0.690 \mu \mathrm{m}$, this relative error of about $+0.6 \%$ in one parameter value can induce a dramatic error in the value of the derivative $\partial I / \partial n_{o}$ : indeed the true value of the derivative will be multiplied by $(-1)$ if measurement is performed at $\theta \approx 31^{\circ}$ and by $(1 / 3)$ if

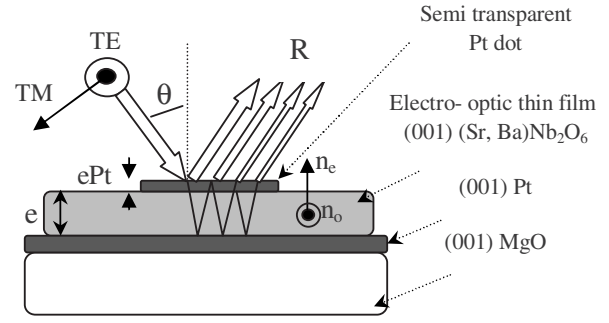

FIG. 3. Fabry-Perot interferometric configuration. The EO film, optically uniaxial (001) SBN in the present work, is sandwiched between two Pt electrodes. The (001) orientation of the underlying Pt electrode is a specific requirement for $(001)$ oriented $\mathrm{SBN}$ growth. Reflectivity $(R)$, and variation in reflectivity $(\Delta R)$ induced by an ac modulating voltage, are recorded vs incident angle $\theta$, successively for transverse electric TE and transverse magnetic TM polarizations

measurement is performed at $\theta \approx 34^{\circ}$. Values of $\Delta n_{o}$ and of the corresponding EO coefficient will consequently be inferred erroneous in the inverse proportions, that is, obtained with the wrong sign if measurement is performed at $\theta$ $\approx 31^{\circ}$, or overestimated by a factor 3 if measurement is performed at $\theta \approx 34^{\circ}$. From this simple demonstration it appears that determination of $\mathrm{EO}$ coefficients is liable to drastic errors and must be necessarily checked independent of angle of incidence. Nevertheless, this independence is not sufficient to ensure right results, as will be shown in the application below.

\section{B. Principle of the present characterization method}

The method presented in this work was developed with the aim of increasing the reliability of EO coefficients determination to a maximum. We have chosen a Fabry-Perot interferometric setup which is the simplest one in terms of both implementation and modeling. It enables determining separately EO coefficients The method exploits interferences obtained by reflection on the stack made of the EO film sandwiched between two electrodes (Fig. 3). Films of strontium barium niobate SBN investigated in this work are (001) oriented, optically uniaxial, with ordinary and extraordinary optical axes, respectively, parallel and perpendicular to the substrate surface. Reflectivity $(R)$ and variation in reflectivity $(\Delta R)$ induced by an ac modulating voltage are recorded versus incident angle $\theta$, successively for TE and transversemagnetic (TM) polarizations. According to the above expression (4)

$$
\Delta R_{\mathrm{TE}, \theta}=\left(\frac{\partial R}{\partial n_{o}}\right)_{\mathrm{TE}, \theta} \Delta n_{o}+\left(\frac{\partial R}{\partial e}\right)_{\mathrm{TE}, \theta} \Delta e+\left(\frac{\partial R}{\partial k_{o}}\right)_{\mathrm{TE}, \theta} \Delta k_{o}
$$

where $\Delta e, \Delta n_{o}$, and $\Delta k_{o}$ are the electric-field-induced variations in, respectively, the active film thickness $e$, its ordinary refractive index $n_{o}$, and its ordinary extinction coefficient $k_{o}$

Calculation of reflectivity $R$ is performed from Fresnel formulae by taking into account all the multireflection effects in the stack. ${ }^{30}$ The value of each derivative is numerically calculated, for instance $\left(\partial R / \partial n_{o}\right)_{\mathrm{TE}, \theta}=\left[R_{\mathrm{TE}, \theta}\left(n_{o}+d n_{o}\right)\right.$ $\left.-R_{\mathrm{TE}, \theta}\left(n_{o}\right)\right] / d n_{o}$, where $d n_{o}$ is a sufficiently small variation in $n_{o}$. Determination of the three unknowns $\Delta n_{o}, \Delta e$, and $\Delta k_{o}$ appearing in (5) is then achieved by selecting three experi- 
mental data $\left[\Delta R_{\mathrm{TE}}\left(\theta_{1}\right), \Delta R_{\mathrm{TE}}\left(\theta_{2}\right), \Delta R_{\mathrm{TE}}\left(\theta_{3}\right)\right]$ and solving the inferred system of three linear equations. At this step, the EO coefficient $r_{13}$, defined in Eq. (1), and the CPE coefficient $d_{33}$ can be deduced

$$
\begin{aligned}
& r_{13}=\frac{-2 e \Delta n_{o}}{n_{o}^{3} \Delta V}, \\
& d_{33}=\frac{\Delta e}{\Delta V},
\end{aligned}
$$

where $\Delta V$ is the ac modulating voltage amplitude. The refractive index of the film seen by TM polarization $\left(n_{\theta}\right)$ and its variation under electric-field $\left(\Delta n_{\theta}\right)$ are dependent on $\theta$, which prevents the use of a similar procedure. If $\theta_{F}$ represents the refracting angle of the light wave in $\mathrm{SBN}, n_{\theta}$ and $\Delta n_{\theta}$ verify

$$
\begin{aligned}
& \frac{1}{n_{\theta}^{2}}=\frac{\cos ^{2} \theta_{F}}{n_{o}^{2}}+\frac{\sin ^{2} \theta_{F}}{n_{e}^{2}}, \\
& n_{\theta}=n_{o} \times\left[1-\sin ^{2} \theta \times\left(\frac{1}{n_{e}^{2}}-\frac{1}{n_{o}^{2}}\right)\right]^{1 / 2}, \\
& \Delta n_{\theta}=-\frac{n_{\theta}^{3} \Delta V}{2 e} \times\left(r_{13} \cos ^{2} \theta_{F}+r_{33} \sin ^{2} \theta_{F}\right) .
\end{aligned}
$$

According to Eq. (4)

$$
\begin{aligned}
\Delta R_{\mathrm{TM}, \theta}= & \left(\frac{\partial R}{\partial n_{\theta}}\right)_{\mathrm{TM}, \theta} \Delta n_{\theta}+\left(\frac{\partial R}{\partial e}\right)_{\mathrm{TM}, \theta} \Delta e \\
& +\left(\frac{\partial R}{\partial k_{\theta}}\right)_{\mathrm{TM}, \theta} \Delta k_{\theta} .
\end{aligned}
$$

Derivatives appearing in Eq. (11) are calculated numerically like they were calculated for TE polarization except that dependence of index on $\theta$ must be taken into account. A value of incident angle is selected for which $\left(\partial R / \partial k_{\theta}\right)_{\mathrm{TM}, \theta}=0$, and Eq. (11) becomes a linear equation with a single unknown $\left(\Delta n_{\theta}\right)$. The EO coefficient $r_{33}$ is deduced from the solution $\Delta n_{\theta}$ through above expression (10).

From values of $r_{13}, d_{33}$, and $\Delta k_{o}$, the electric-field induced response $\Delta R_{\mathrm{TE}}(\theta)$ may be calculated versus incident angle and compared to the experimental data. Calculation must account for both experimental $R_{\mathrm{TE}}(\theta)$ and $\Delta R_{\mathrm{TE}}(\theta)$. When it does not, an error in the value of at least one parameter of the stack is the most likely reason and determination of film electric-field induced response is then liable to drastic errors, as illustrated above. When calculation accounts for the whole of TE data, the uniqueness of the solution may be checked as will be shown in the application below. For TM polarization, calculation of $R_{\mathrm{TM}}(\theta)$ and $\Delta R_{\mathrm{TM}}(\theta)$ versus incident angle may be carried out by considering $n_{e}$ as a parameter, provided the approximations $k_{\theta} \approx k_{o}$ and $\Delta k_{\theta} \approx \Delta k_{o}$ are used. These approximations may cause a discrepancy between calculation and experiment at large incident angles. This possible discrepancy excepted, calculation must be checked to account for the whole of experimental data: $R_{\mathrm{TE}}(\theta), R_{\mathrm{TM}}(\theta), \Delta R_{\mathrm{TE}}(\theta)$, and $\Delta R_{\mathrm{TM}}(\theta)$.

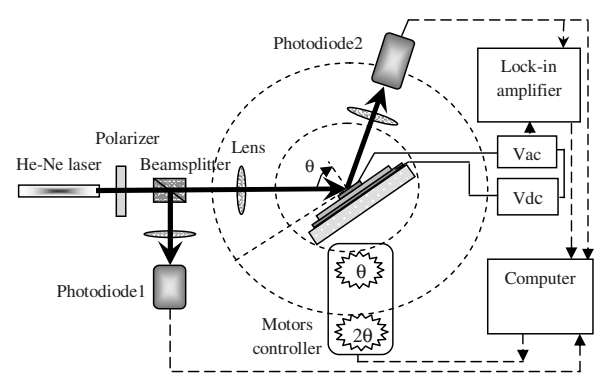

(a)

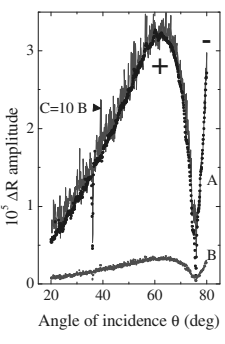

(b)
FIG. 4. (a) Experimental setup used for electric-field-induced response characterization. (b) Example of variations in TM reflectivity (amplitude and sign) measured vs incident angle when applying a modulated voltage $V_{\text {ac }}$ either $1 \mathrm{~V}$ (dotted A) or $0.1 \mathrm{~V}$ (dotted B) in amplitude. The latter is also multiplied by 10 in the figure (gray continuous line $\mathrm{C}$ ) in order to check that response is proportional to applied voltage.

\section{Experimental setup}

Measurements of $R_{\mathrm{TE}}(\theta), \quad R_{\mathrm{TM}}(\theta), \quad \Delta R_{\mathrm{TE}}(\theta), \quad$ and $\Delta R_{\mathrm{TM}}(\theta)$ are performed by using the simple $\theta-2 \theta$ goniometric setup schematically represented in Fig. 4(a). The sample is fixed on the central rotating part so that the plane of incidence is horizontal and the position of the light spot, centered on a platinum dot, is independent of incident angle. Light is emitted by a He-Ne laser operating at $633 \mathrm{~nm}$, polarized either perpendicular (TE) or parallel (TM) to the sample plane of incidence, then split in two perpendicular beams. Intensities of the beams reflected by the beam splitter and by the sample are measured simultaneously by two silicon photodiodes and the absolute value of sample reflectivity is inferred. Electric-field applied to EO film is made of both a dc part, which ensures a constant poling, and an ac part, which modulates reflectivity at a low frequency about 230 Hz. A lock-in amplifier detects amplitude and phase of the modulated reflectivity $\Delta R(\theta)$. An example of experimental data is given in Fig. 4(b): the amplitude of $\Delta R(\theta)$ is plotted versus incident angle and its sign, provided by phase measurement, is noted down. As depicted in the figure, $\Delta R(\theta)$ is preliminary checked to be a linear response proportional to the applied ac voltage.

\section{STRONTIUM BARIUM NIOBATE EPITAXIAL THIN FILMS}

Crystalline $\mathrm{Sr}_{\mathrm{x}} \mathrm{Ba}_{1-\mathrm{x}} \mathrm{Nb}_{2} \mathrm{O}_{6} \quad(\mathrm{SBN}: \mathrm{x}, 25 \%<\mathrm{x}<75 \%)$ is a tungsten bronze ferroelectric solid solution which has been widely studied for holographic recording and optical processing. Increasing strontium relative content $(\mathrm{x})$ reduces crystalline cell parameters and lowers Curie temperature which results in an enhancement of most material properties measured at room temperature. At $\lambda=633 \mathrm{~nm}$ and room temperature under static field, the value of the linear EO coefficient $r_{33}$ increases from $230 \mathrm{pm} / \mathrm{V}$ (Ref. 31) to 1340 $\mathrm{pm} / \mathrm{V}$ (Ref. 32) when $\mathrm{x}$ varies from $60 \%$ to $75 \%$. The latter is the largest known $r_{33}$ value, nearly two orders of magnitude larger than that of the primary $\mathrm{EO}$ material $\mathrm{LiNbO}_{3}$. Although the dielectric permittivity of SBN also reaches values about two orders of magnitude larger than that of LN and because EO coefficient is involved to the power 2 in expression (3) of drive power, replacing LN by SBN divides this 
(a)

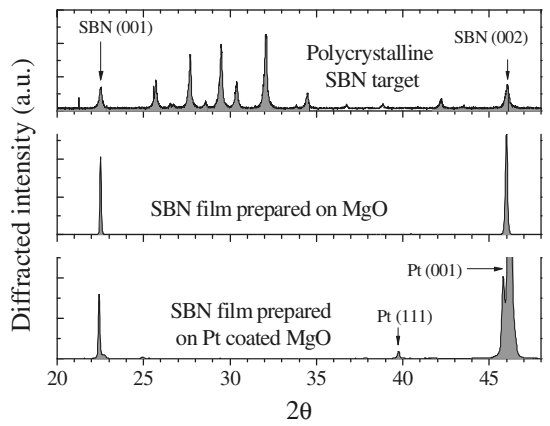

(b)

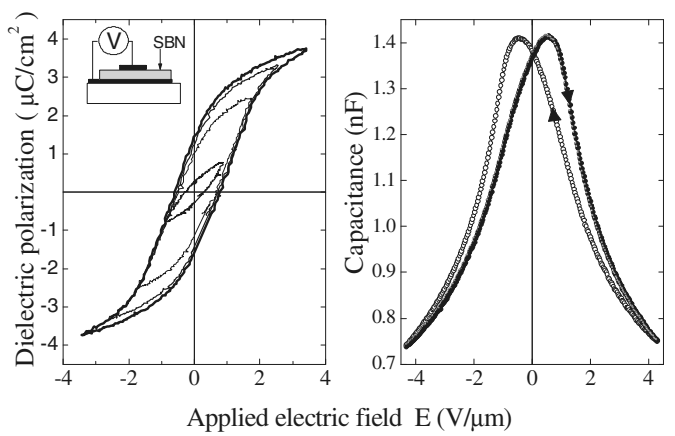

FIG. 5. Typical structural and dielectric properties of SBN films we prepare by using rf sputtering deposition followed by a rapid thermal crystallization. (a) x-ray diffraction patterns of two SBN films prepared on $\mathrm{MgO}$ and Pt coated $\mathrm{MgO}$ demonstrating films (001) orientation. The pattern of a polycrystalline target used for sputtering is given for reference. (b) Ferroelectric polarization-electric-field hysteresis loops recorded at a frequency of $33 \mathrm{~Hz}$ and room temperature with increasing electric-field modulation amplitude. (c) Electric-field dependence of the weak field capacitance of a Pt/SBN/Pt structure at $10 \mathrm{kHz}$ and room temperature. Arrows indicate the input voltage sweep direction. SBN films exhibit a strong dielectric non linearity: $\left[\varepsilon_{3}(E)-\varepsilon_{3}(0)\right] / \varepsilon_{3}(0) \approx-46 \%$ for $E$ $\approx 4 \mathrm{~V} / \mu \mathrm{m}$.

drive power by a factor varying from about 3 when $\mathrm{x}$ $=60 \%$ to about 20 when $x=75 \%$. Thin films of SBN are, therefore, particularly attractive for their potential use as low voltage EO waveguides.

Implementation of SBN EO properties requires a successful heteroepitaxial growth of the film with (001) SBN direction perpendicular to a lattice-matched conductive substrate plane. We have achieved the (001)SBN\|(001)Pt $\|$ (001) MgO double epitaxial growth by using RF magnetron sputtering of $\mathrm{Pt}$ and ceramic SBN targets. Parameters of $\mathrm{Pt}$ and SBN deposition have been fixed from the results of previous investigations in which we established conditions for a stoichiometric composition of the amorphous SBN deposit ${ }^{33}$ and for an epitaxial crystallization of a single phase SBN on Pt covered $\mathrm{MgO}$ substrates. ${ }^{34,35}$ Amorphous SBN films are crystallized in a rapid thermal annealing furnace at $1160{ }^{\circ} \mathrm{C}$ during a few seconds. The (001) oriented growth of SBN on Pt occurs exclusively on (001) oriented Pt. ${ }^{34}$ The x-ray diffraction phi-scans we measured from (220) Pt planes and (211) and (311) SBN planes have demonstrated that in-plane orientations of SBN are mirror symmetric $\left( \pm 18^{\circ}\right)$ to the cell axes of (001) Pt and (001) MgO. ${ }^{34}$ Top platinum dots deposited on the surface enable dielectric and EO characterizations. When films are prepared in the optimized deposition and crystallization conditions, they exhibit ferroelectric properties and a strong dielectric non linearity at room temperature. ${ }^{35}$ A relative variation in dielectric permittivity $\left[\varepsilon_{3}(E)-\varepsilon_{3}(0)\right] / \varepsilon_{3}(0)$ of $46 \%$ may be obtained by applying an electric-field $\mathrm{E}$ of about $4 \mathrm{~V} / \mu \mathrm{m}$. Some typical properties of the SBN films we have developed are summarized in Fig. 5.

The initial approximate knowledge of SBN and Pt layer thicknesses in the Fabry-Perot stack is based on measurements we previously performed on $\mathrm{SBN} / \mathrm{MgO}$ and $\mathrm{Pt} / \mathrm{MgO}$ separate samples in order to determine deposition rates versus deposition conditions. Normal transmission measurements versus wavelength in the IR-visible spectral range were used for SBN/MgO samples and grazing incidence $\mathrm{X}$-ray reflectivity measurements ${ }^{36}$ at the $\mathrm{x}$-ray wavelength of $0.154 \mathrm{~nm}$ were used for $\mathrm{Pt} / \mathrm{MgO}$ samples. By fitting a calculated reflectivity to that measured on $\mathrm{Pt} / \mathrm{MgO}$ versus incident angle at $\lambda=633 \mathrm{~nm}$, we checked that complex index reported in tables for Pt (see Table I) accounted for Pt film reflectivity. In samples used for EO characterization, $\mathrm{SBN}$ is deposited on $\mathrm{Pt}$ and not on $\mathrm{MgO}$, which could modify its deposition rate as well as its density and complex index. Moreover the stress of SBN is different when crystallized alone on $\mathrm{MgO}$ or when inserted in a stack, which could also modify film parameters. For all these reasons, the knowledge of some of the stack parameter values is necessarily initially approximate.

When crystallized on platinum, SBN films exhibit a rough surface. Taking this roughness into account systematically yielded discrepancies between experimental and calculated electric-field-induced responses. Although our modeling of interface roughness allowed accounting satisfactorily for reflectivity data, it did not for the electric-field-induced variation in reflectivity. The above underlined sensitivity of reflectivity derivatives to a slight discrepancy between model and sample is the reason for this failure, and the imperfection of roughness modeling is a strong source of potential error for the electric-field-induced response characterization. Therefore, the surface of the SBN film investigated in this work was mechanically polished before deposition of top platinum dots. The average roughness of bottom Pt surface was measured close to $7 \AA$, and that of SBN surface after polishing was measured close to $16 \AA$, by using a profilometer (Dektak 8). The size of Pt top dots must be larger than that of the light spot in a sufficiently broad range of incident

TABLE I. Parameter values for substrate and layers in Fig. 3 which allow accounting for both experimental reflectivity $R$ and electric-field-induced variation in reflectivity $\Delta R$, vs incident angle, for both TE and TM polarizations.

\begin{tabular}{lccc}
\hline \hline Layer & $\begin{array}{c}\text { Thickness } \\
(\AA)\end{array}$ & $\begin{array}{c}\text { Refractive index } \\
(\lambda=633 \mathrm{~nm})\end{array}$ & $\begin{array}{c}\text { Extinction coefficient } \\
(\lambda=633 \mathrm{~nm})\end{array}$ \\
\hline MgO substrate $^{\mathrm{a}}$ & $500 \times 10^{4}$ & 1.7346 & 0 \\
${\text { Bottom } \mathrm{Pt}^{\mathrm{a}}}_{\text {SBN:60 }}^{700}$ & 2.33 & 4.14 \\
Top Pt dot & $e=7545$ & $n_{o}=2.30, n_{e}=2.26$ & $k_{o}=0.0515$ \\
& $e \mathrm{Pt}=226$ & 2.33 & 4.14 \\
\hline
\end{tabular}

${ }^{\mathrm{a} C o m p l e x}$ index from Ref. 49. 
angles without increasing excessively the probability of a short-circuit between electrodes. The following results have been obtained with a top Pt circular dot $1.5 \mathrm{~mm}$ in diameter.

\section{RESULTS}

\section{A. Reflectivity fitting procedure}

We have underlined above the decisive role of an accurate knowledge of parameter values. Reflectivity fitting procedures enable refining this initially approximate knowledge. A calculated TE reflectivity was successively fitted to the reflectivity measured on SBN surface between two Pt dots, then to that measured on the selected Pt dot $\left(R_{\mathrm{TE}}\right)$. The first fitting procedure did not involve Pt dot parameters and allowed determining a limited range of possible values for each of the three SBN parameters $\left(e, n_{o}, k_{o}\right)$. The knowledge of only one of these three parameters was necessary to continue and we selected the ordinary refractive index $n_{o}$ which is less liable to variation than film thickness when moving light spot to a $\mathrm{Pt}$ dot center. The second fitting procedure $\left(R_{\mathrm{TE}}\right)$ was, therefore, carried out by assuming $n_{o}$ $=2.30 \pm 0.01$, and aimed at refining values of the three approximately known parameters: $e, k_{o}$, and Pt dot thickness $e$ Pt. Figure 6 illustrates this second fitting procedure: successive values of $e \mathrm{Pt}$ have been considered in the neighborhood of the value expected from calibration, and, for each one, isoreflectivity curves $R_{\mathrm{TE}, \theta}\left(e, k_{o}\right)$ have been calculated from a few measured values of TE reflectivity at different angles of incidence. If the different iso- $R_{\mathrm{TE}, \theta}$ curves cut in a common $\left(e, k_{o}\right)$ intersection [Fig. 6(a)], a fitting set of parameters exists and is thus determined. Similar iso- $R_{\mathrm{TM}, \theta}$ curves can be plotted for TM polarization [Fig. 6(b)] provided we neglect the variation in SBN index with incident angle caused by expected birefringence. This approximation did not affect significantly the convergence of iso- $R_{\mathrm{TM}, \theta}$ curves at the chosen incident angles [Fig. 6(b)], and consistent $(e, k)$ intersections were obtained for TE and TM polarizations. Different fitting sets of parameters were thus determined. Reflectivities calculated from the three fitting sets of parameters determined in Fig. 6(a) have been plotted in Fig. 6(c), where they may be compared to experimental data. The comparison for TM polarization should allow eliminating some of the fitting solutions provided large values of incident angle $\left(>75^{\circ}\right)$ are considered. But a reliable measurement of reflectivity is practically impossible at large incident angles because the size of the light spot exceeds that of the Pt dot, thus causing the drop observed in measured reflectivity. At small incident angles, measurements are hindered by the unavoidable shadow cast by electric contact, and particularly by the electric wire around $\theta=36^{\circ}$.

The evolution of the common $\left(e, k_{o}\right)$ intersection of iso- $R_{\mathrm{TE}}$ with the assumed value of $\mathrm{Pt}$ dot thickness is represented in Fig. 7. The uncertainty affecting $n_{o}$ has been taken into account and the fitting sets of parameters have been determined also in the two extreme cases $n_{o}=2.29$ and $n_{o}$ $=2.31$. We can verify that a slight error in a parameter value is able to yield largely erroneous results. Indeed, if we consider the three different fitting sets of parameters represented by dots A, B, and C in Fig. 7, and carry out the character- (a)

(b)
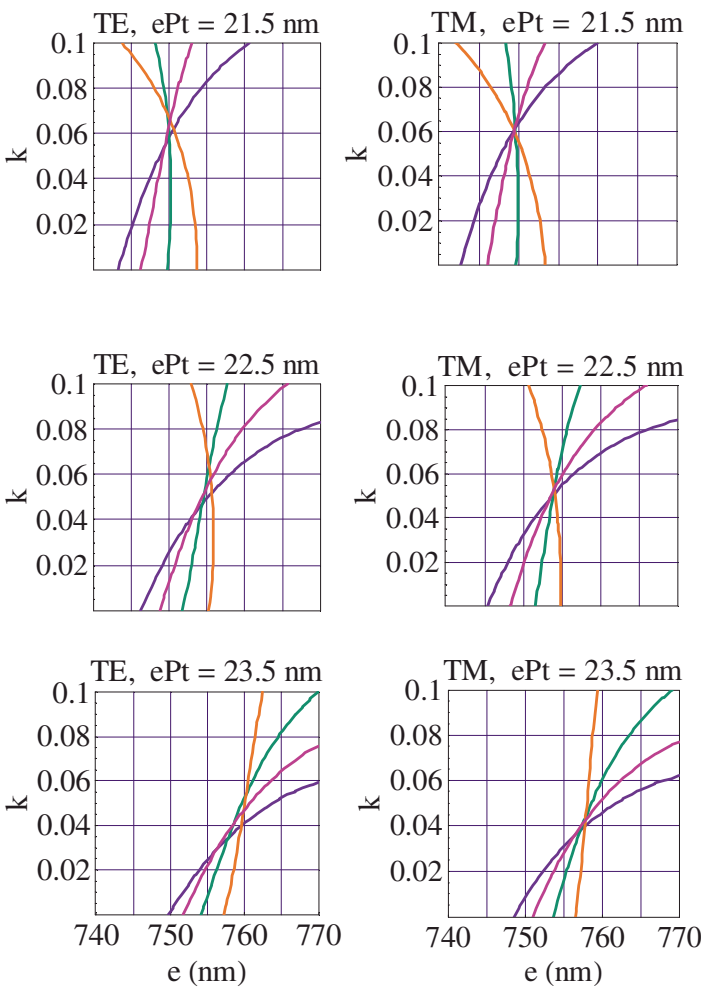

(c)

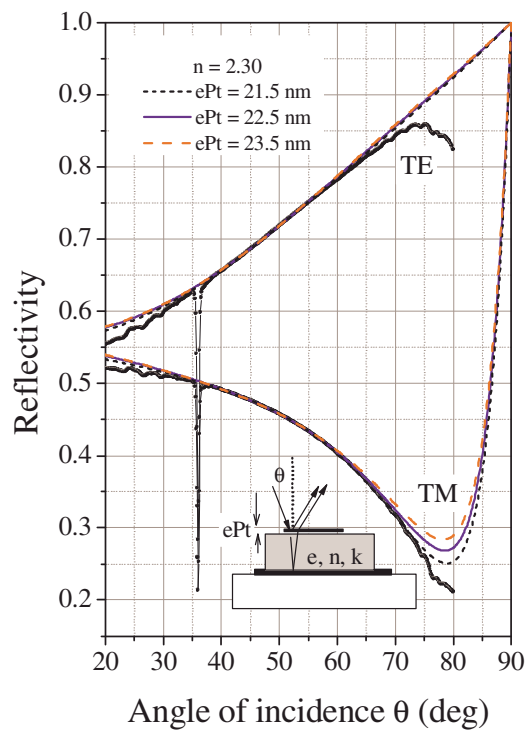

FIG. 6. (Color online) [(a) and (b)] Isoreflectivity curves $R_{\mathrm{TE}, \theta}(e, k)(\mathrm{a})$ and $R_{\mathrm{TM}, \theta}(e, k)$ (b). Iso- $R$ are calculated for experimental values of TE and TM reflectivity measured at various angles of incidence $(\theta$ $\left.=40^{\circ}, 45^{\circ}, 50^{\circ}, 55^{\circ}\right)$ on a Pt/SBN/Pt structure. The two variables $(e, k)$ are SBN film thickness and extinction coefficient. Three different possible values of the top Pt electrode thickness ePt are considered close to the value expected from calibration: $e \mathrm{Pt}=21.5,22.5,23.5 \mathrm{~nm}$. Calculation is carried out by using values beforehand determined for parameters other than $(e \mathrm{Pt}, e, k)$ (Table I). For TM polarization, variation in $\mathrm{SBN}$ index with incident angle is neglected and SBN refractive index is taken to be $n=2.30$. If the different iso- $R$ curves cut in a common $(e, k)$ point of intersection, a fitting set of parameters exists and is thus determined. (c) Reflectivities calculated with the three fitting sets of parameters determined from the points of intersection in (a) and (b). Dotted lines depict measured TE and $\mathrm{TM}$ reflectivities. 


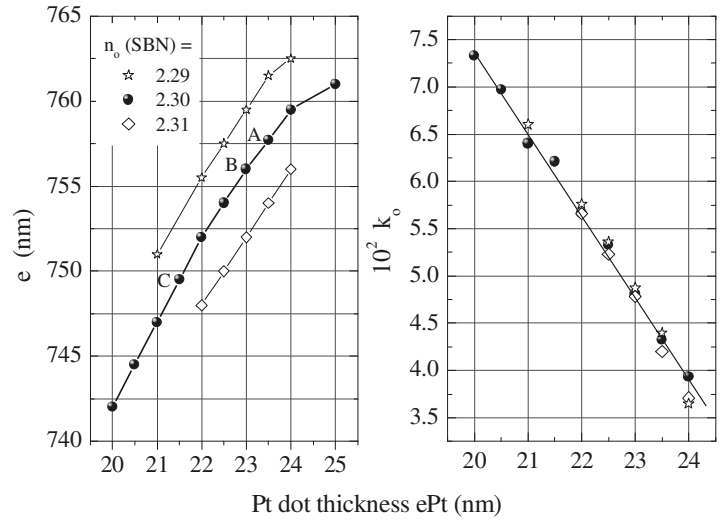

FIG. 7. Evolution of the common $\left(e, k_{o}\right)$ point of intersection of iso- $R_{\mathrm{TE}}$ curves with the assumed value of $\mathrm{Pt}$ dot thickness; $\left(e, k_{o}\right)$ are $\mathrm{SBN}$ film thickness and ordinary extinction coefficient. The uncertainty affecting SBN ordinary refractive index $n_{o}$ has been taken into account and fitting sets of parameters have been determined also in the two extreme cases $n_{o}=2.29$ and $n_{o}=2.31$. Dots A, B, and C specify three particular sets of parameters among those which allow accounting for experimental reflectivity.

ization of electric-field induced response for TE polarization as detailed in Sec. II B, we obtain EO coefficients as different as $r_{13}=-41 \mathrm{pm} / \mathrm{V}(\mathrm{A}),+30 \mathrm{pm} / \mathrm{V}(\mathrm{B}),-1.5 \mathrm{pm} / \mathrm{V}(\mathrm{C})$. The corresponding three calculated responses $\Delta R_{\mathrm{TE}, \theta}$ are displayed in Fig. 8(b). It is to be noted that the strong difference obtained between calculated responses $\mathrm{A}$ and $\mathrm{B}$ is caused only by a $5 \AA$ variation in the assumed value of Pt dot thickness. As expected, all calculated responses coincide with experimental data when incident angle identifies with one of the angles $\left(\theta_{1}, \theta_{2}, \theta_{3}\right)$ selected to build the linear system of Eqs. (5). On the other hand, none of them fits experimental data except in a short range of angles. This indicates that all three are erroneous. Moreover the chaotic evolution of the calculated response with the assumed value of $e \mathrm{Pt}$ is of no help to approach the right set of parameters.

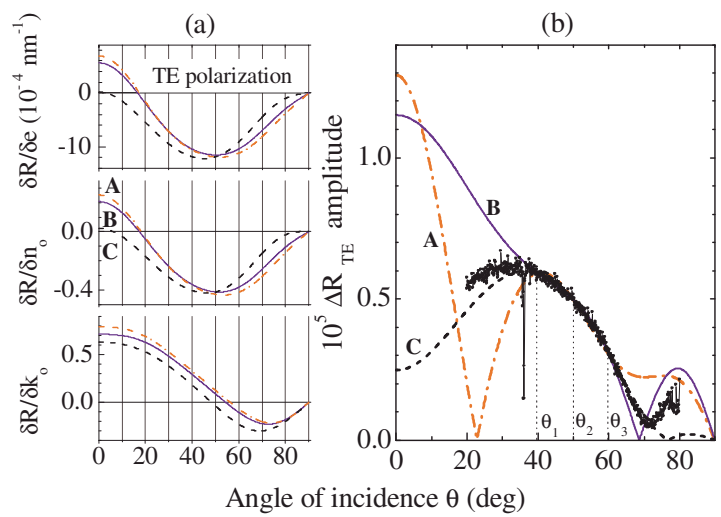

FIG. 8. (Color online) (a) Derivatives $\partial R / \partial e, \partial R / \partial n_{o}$, and $\partial R / \partial k_{o}$ calculated for TE polarization from the three parameter sets corresponding to dots $\mathrm{A}$, $\mathrm{B}$, and $\mathrm{C}$ in Fig. 7. (b) Responses $\Delta R_{\mathrm{TE}, \theta}$, respectively labeled $\mathrm{A}, \mathrm{B}$, and $\mathrm{C}$, calculated from the results of the characterization procedure when using these three different parameter sets. The dotted line depicts $\Delta R_{\mathrm{TE}, \theta}$ amplitude measured with an applied modulated voltage of $1 \mathrm{~V}$ amplitude. Experimental data used to carry out characterization are $\Delta R_{\mathrm{TE}}\left(\theta_{1}\right), \Delta R_{\mathrm{TE}}\left(\theta_{2}\right)$, and $\Delta R_{\mathrm{TE}}\left(\theta_{3}\right)$. The EO coefficient $r_{13}$ is determined to be equal to $-41,+30$, or $-1.5 \mathrm{pm} / \mathrm{V}$ when using parameter sets $\mathrm{A}, \mathrm{B}$, or $\mathrm{C}$, respectively. The piezoelectric coefficient $d_{33}$ is simultaneously determined to be equal to -126 $\mathrm{pm} / \mathrm{V}(\mathrm{a}),+85 \mathrm{pm} / \mathrm{V}$ (b), or $-9 \mathrm{pm} / \mathrm{V}$ (c). None of these three parameter sets allows accounting for experimental $\Delta R_{\mathrm{TE}}(\theta)$ in the full range of incident angles.

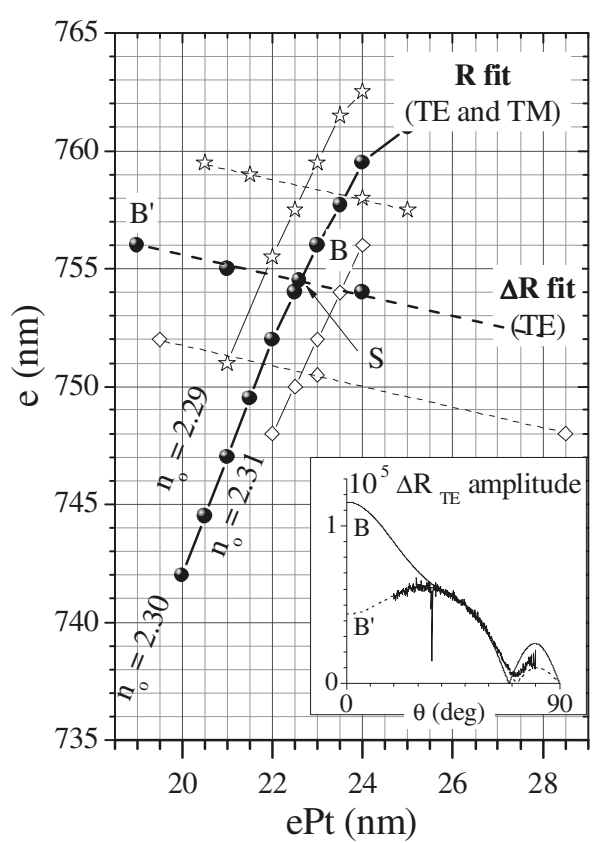

FIG. 9. Couples Pt dot thickness-SBN film thickness $(e \mathrm{Pt}, e)$ which allow fitting either calculated reflectivities to experimental ones (" $R$ fit"continuous line) or a calculated variation in TE reflectivity to experimental one ( $\Delta R$ fit-dashed line). An example is given in the inset: $\Delta R_{\mathrm{TE}}$ is calculated from the parameter sets associated with either $\operatorname{dot} \mathrm{B}$ or $\operatorname{dot} \mathrm{B}^{\prime}$ and compared to experimental $\Delta R_{\mathrm{TE}}$. The set of parameters which allows accounting for both TE reflectivity and electric-field-induced variation in TE reflectivity may be graphically determined and is unique (dot $\mathrm{S}$ for $n_{o}$ $=2.30$ ). Uncertainty affecting the film refractive index has been taken into account and the two possible extreme cases $\left(n_{o}=2.29\right.$ and $\left.n_{o}=2.31\right)$ are also represented in the figure.

\section{B. Electric-field-induced response fitting procedure (TE polarization)}

Given one of the previous fitting sets of parameters (symbol B in Fig. 7 and Fig. 9 for example), if we give up accounting for reflectivity data temporarily and attempt to fit $\Delta R_{\mathrm{TE}, \theta}$ by adjusting the crucial value of $\mathrm{Pt}$ dot thickness, we find a second set of parameters (symbol B' in Fig. 9) which allows fitting $\Delta R_{\mathrm{TE}, \theta}$ (see inset in Fig. 9). The evolution of this second fitting value of $e \mathrm{Pt}$ with the chosen first set of parameters is represented in Fig. 9 with a dashed line labeled " $\Delta R_{\mathrm{TE}}$ fit." Again multiple solutions were found, which all account for experimental $\Delta R_{\mathrm{TE}, \theta}$. It is therefore to be noted that, even though it is a necessary condition, the fact that a calculated response $\Delta R_{\mathrm{TE}, \theta}$ fits experimental data in the whole range of incident angles is not sufficient to ensure reliable results.

Given a value of the refractive index $n_{o}$, a single set of parameters allows accounting for both TE reflectivity and electric-field-induced variation in TE reflectivity: it may be graphically determined and is unique (dot $\mathrm{S}$ in Fig. 9). The characterization procedure described in Sec. II B was carried out from this set of parameters detailed in Table I, and yielded the results indicated below [Eq. (12)]. EO, CPE, and EA components calculated from Eq. (12) are shown in Fig. 10 . The uncertainty affecting $n_{o}$ does not modify significantly the graphically determined value of $e \mathrm{Pt}$ (see Fig. 9) nor the correlated value of $k_{o}$ [see Fig. 7(b)], and it finally induces the relatively low uncertainties indicated below 
(a)

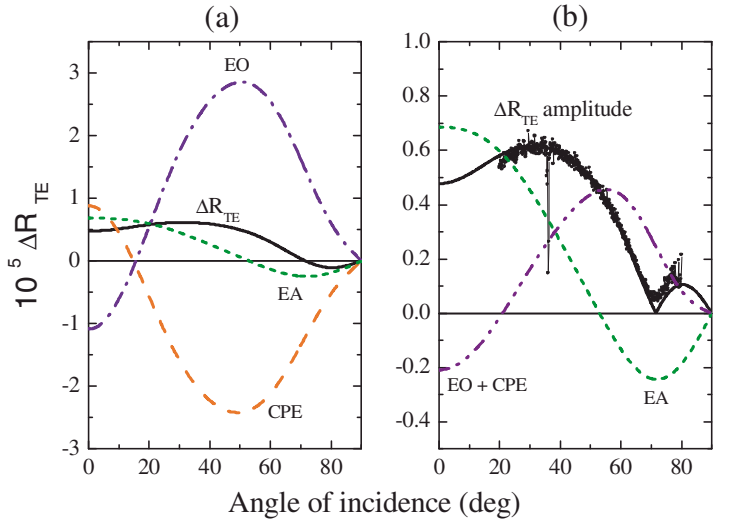

FIG. 10. (Color online) (a) EO, CPE, and EA components of the electricfield induced variation in TE reflectivity $\left(\Delta R_{\mathrm{TE}}\right)$ calculated from characterization procedure results. (b) Comparison between calculated (continuous line) and experimental (dotted line) $\Delta R_{\mathrm{TE}}$ amplitudes. Amplitude of modulated voltage applied to SBN film is $1 \mathrm{~V}$. The plot of (EO+CPE) and (EA) components underlines the significant contribution of EA effect to total TE response.

$$
\begin{aligned}
& r_{13}=+8.5 \pm 1.3 \mathrm{pm} / \mathrm{V}, \\
& d_{33}=\Delta e / \Delta V=+21 \pm 4 \mathrm{pm} / \mathrm{V}, \\
& \Delta k_{o} / \Delta V=(+9.8 \pm 0.6) \times 10^{-6} .
\end{aligned}
$$

These results are therefore the single ones compatible with both experimental reflectivity and TE variation in reflectivity versus incident angle. The value of $r_{13}$ is very different from each of those above obtained when this double requirement was not fulfilled (Sec. IV A). As may be noticed in Fig. 10, none of the three contributions is negligible and any attempt to account for experimental data without considering all of them would have been necessarily doomed to failure. Due to the opposite signs of $\Delta n_{o}$ and $\Delta e$, phase shifts induced by EO and CPE effects partially compensate one another so that the sum of these two contributions [Fig. 10(b)] does not prevail over the EA component in the final signal $\Delta R_{\mathrm{TE}, \theta}$. A similar compensation is to be expected in a waveguide configuration if similarly low modulating frequencies are used.

\section{Electric-field-induced response fitting procedure (TM)}

The refractive index of SBN seen by polarization TM $\left(n_{\theta}\right)$ and its variation under electric-field $\left(\Delta n_{\theta}\right)$ are dependent on incident angle. Following the procedure detailed in Sec. II B, we calculated the reflectivity derivatives for TM polarization from the right set of parameters in Table I, by using Eq. (9) where the extraordinary index $n_{e}$ is a varied parameter. Equations (10) and (11) were then written by considering an incident angle $\theta k$ for which the value of the derivative $(\partial R / \partial k)_{\mathrm{TM}, \theta}$ is zero. This allowed eliminating the unknown $\Delta k_{\theta}$ from Eq. (11) and extracting the value of $r_{33}$ from the following equation:
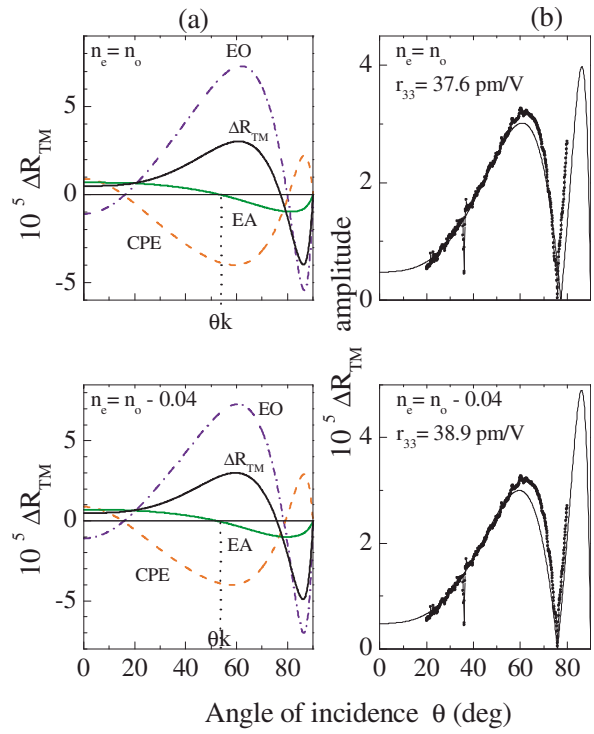

FIG. 11. (Color online) Electric-field induced response for TM polarization $\left(\Delta R_{\mathrm{TM}}\right)$ calculated from characterization procedure results by assuming $k_{\theta}$ $\approx k_{o}, \Delta k_{\theta} \approx \Delta k_{o}$ ( $k$ represents SBN film extinction coefficient). The three $\mathrm{EO}, \mathrm{CPE}$, and EA contributions are detailed in (a) and comparison with experimental response is given in (b). Amplitude of modulated voltage applied to SBN film is $1 \mathrm{~V}$. Extraordinary refractive index $n_{e}$ is the single adjustable parameter of characterization procedure; two cases are shown: $n_{e}=n_{o}$ (top) and $n_{e}=n_{o}-0.04$ (bottom). The latter corresponds to the best calculation-experiment fit. The EO coefficient $r_{33}$ determined from characterization procedure and used for $\Delta R_{\mathrm{TM}}$ calculation is equal to +37.6 (top) and $+38.9 \mathrm{pm} / \mathrm{V}$ (bottom).

$$
\begin{gathered}
-\frac{n_{\theta k}^{3} \Delta V}{2 e} \times\left(r_{13} \cos ^{2} \theta k_{F}+r_{33} \sin ^{2} \theta k_{F}\right) \\
=\frac{\Delta R_{\mathrm{TM}, \theta k}-\left(\frac{\partial R}{\partial e}\right)_{\mathrm{TM}, \theta k} \times \Delta e}{\left(\frac{\partial R}{\partial n_{\theta}}\right)_{\mathrm{TM}, \theta k}},
\end{gathered}
$$

where $\theta k_{F}$ represents the refracting angle of the light wave in $\mathrm{SBN}$. Electric-field induced response in TM polarization $\Delta R_{\mathrm{TM}, \theta}$ can be calculated from this last result and compared to the experimental data, provided we neglect the possible variations in $k_{\theta}$ and $\Delta k_{\theta}$ with incident angle $\left(k_{\theta} \approx k_{o}, \Delta k_{\theta}\right.$ $\approx \Delta k_{o}$ ). This last approximation may cause a discrepancy at large incident angles. Figure 11 shows that a satisfactory agreement is obtained, which may be improved by varying the single adjustable parameter of this calculation: $n_{e}$. The resulting value of $n_{e}\left(n_{e}=n_{o}-0.04\right)$ is consistent with the birefringence value reported in the literature for crystalline SBN of similar composition at $\lambda=633 \mathrm{~nm}\left(n_{e} \approx n_{o}-\right.$ 0.03) ${ }^{37}$ Due to compensation effects in Eq. (13), the relative uncertainty affecting $r_{33}$ is lower than that affecting $r_{13}$ or $d_{33}$ and the final result is

$$
\begin{aligned}
& r_{33}=+38.9 \pm 0.5 \mathrm{pm} / \mathrm{V}, \\
& \frac{r_{33}}{r_{13}}=+4.5 \pm 0.6 .
\end{aligned}
$$

As summarized in Fig. 12, the values obtained for the parameters of the stack and for the coefficients involved in 


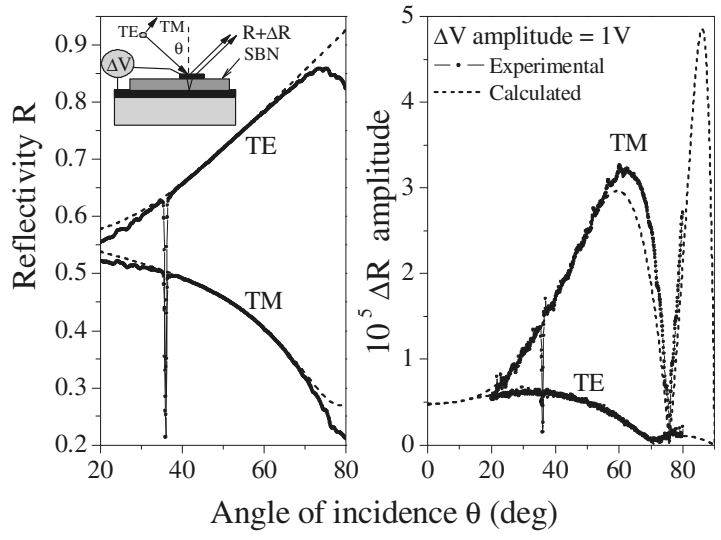

FIG. 12. Summary: reflectivity and electric-field-induced variation in reflectivity of a Pt/SBN/Pt structure for TE and TM polarizations. Calculated and experimental data are compared. Calculation was carried out by using parameter values given in Table I. Electric-field-induced responses were calculated by using the results obtained in the present work: $r_{13}=+8.5 \mathrm{pm} / \mathrm{V}$, $r_{33}=+38.9 \mathrm{pm} / \mathrm{V}, d_{33}=+21 \mathrm{pm} / \mathrm{V}$, and $\Delta k_{o} / \Delta V=+9.8 \times 10^{-6}$.

the electric-field-induced response allow accounting for all the experimental data: reflectivity and electric-field-induced variation in reflectivity versus incident angle, for both TE and TM polarizations.

For TM polarization also the CPE contribution appears to be non negligible at the low frequency used for lock-in detection, as may be seen in Fig. 11(a). Measurements are therefore performed in unclamped conditions and the elastooptic effect is likely responsible for a part of the measured coefficients. Assuming that values of elasto-optic coefficients given in the literature ${ }^{38}$ for crystalline SBN:0.61 ( $p_{13}^{E}$ $\left.=0.08, p_{33}^{E}=0.47\right)$ are valid, elasto-optic parts in $r_{13}$ and $r_{33}$ may be inferred from the following relationships: $r_{13}=r_{13}^{S}$ $+p_{13}^{E} \times d_{33}$ and $r_{33}=r_{33}^{S}+p_{33}^{E} \times d_{33}$, where the superscripts $S$ and $E$ mean, respectively, constant strain and constant field. The elasto-optic parts in $r_{13}$ and $r_{33}$ may thus be assessed to be, respectively, $1.7 \mathrm{pm} / \mathrm{V}$ and $9.9 \mathrm{pm} / \mathrm{V}$, that is about $20 \%$ and $25 \%$ of the respective total values. These percentages are close to those experimentally demonstrated in SBN crystals. ${ }^{39}$
At high frequencies above piezoelectric resonance which are used in information optical processing, the CPE effect is clamped and cannot counteract the EO performance by inducing phase shift compensation as in the above measurements. Moreover the dielectric permittivity $\varepsilon_{3}$ involved in drive power $P$ should be reduced (it is reduced by $39 \%$ in SBN crystals ${ }^{39}$ ). On the other hand the elasto-optic part should be subtracted from the above values of EO coefficients (this elasto-optic part represents about 25\% in SBN crystals $^{39}$ ). If the reduction percentages reported for SBN crystals are valid for SBN thin films, values of $V_{\pi} L$ and $P$ expected for SBN thin films at low frequencies, according to Eqs. (2) and (3), should be multiplied at high frequencies by 1.33 and 1.08 , respectively.

\section{DISCUSSION}

A few groups reported on the linear EO effect characterization in their SBN films. ${ }^{11-14,40,41}$ In three of these papers, ${ }^{11,40,41}$ a polarimetric method working in transmission under normal incidence was used, which does not involve $r_{33}$ in the electric-field-induced response. The other reported results are given in Table II. Although the principle of their method enabled a separate measurement of $r_{13}$ by using TE polarization, Trivedi et al. did not exploit this possibility and used the assumption $r_{33} / r_{13}=5$ to determine the two coefficients from one TM measurement. This assumption is common to all the quoted authors and based on one paper ${ }^{42}$ reporting this ratio value for a SBN:60 single crystal at $\lambda$ $=514.5 \mathrm{~nm}$. When measured similarly in a single SBN:60 crystal but at wavelength $\lambda=633 \mathrm{~nm}$, this ratio was reported ${ }^{31}$ to be of a different value: $r_{33} / r_{13}=6.4$. By mistake, Trivedi et al. exchanged $\cos ^{2} \theta_{F}$ and $\sin ^{2} \theta_{F}$ in the expression of $\Delta n_{\theta}$. When correcting this error, their calculation yields $r_{13}=140 \mathrm{pm} / \mathrm{V}$ and $r_{33}=700 \mathrm{pm} / \mathrm{V}$ instead of the values given in Table II. As mentioned above (Sec. II A), the first source of potential error in an EO characterization procedure is an imperfect modeling caused by oversimplifying approximations. Multiple reflections in the film, CPE and EA effects were neglected in the quoted reports. The second

TABLE II. Linear EO coefficients reported in the literature for SBN:60 thin films. Effective coefficient $r_{e f f}=r_{33}-\left(n_{o} / n_{e}\right)^{3} r_{13}$. Results in italics were obtained by assuming a relationship $r_{33} / r_{13}=5$ previously reported (Ref. 42) for a single SBN:60 crystal at $\lambda=514.5 \mathrm{~nm}$.

\begin{tabular}{|c|c|c|c|c|c|c|}
\hline Reference & $\begin{array}{c}\lambda \\
(\mathrm{nm})\end{array}$ & $\begin{array}{c}r_{e f f} \\
(\mathrm{pm} / \mathrm{V})\end{array}$ & $\begin{array}{c}r_{13} \\
(\mathrm{pm} / \mathrm{V})\end{array}$ & $\begin{array}{c}r_{33} \\
(\mathrm{pm} / \mathrm{V})\end{array}$ & $\begin{array}{c}\text { Preparation technique// } \\
\text { substrate }\end{array}$ & $\begin{array}{c}\text { Characterization method } \\
\text { features }\end{array}$ \\
\hline Trivedi $e t a l^{\mathrm{a}}$ & 549 & & 70 & 350 & $\begin{array}{l}\text { Pulsed laser deposition// } \\
\qquad \mathrm{MgO}(001)\end{array}$ & $\begin{array}{c}\text { Interferometric (TM) } \\
\text { Diffraction } \\
\text { Planar electrodes }\end{array}$ \\
\hline Koo et al. ${ }^{\mathrm{b}}$ & 633 & 138.7 & 34.7 & 173.4 & $\begin{array}{c}\text { Sol-gel process// } \\
\mathrm{Pt}(001) / \mathrm{MgO}(001)\end{array}$ & $\begin{array}{c}\text { Polarimetric } \\
\text { Reflection } \\
\text { Sandwich configuration } \\
\text { Polarimetric }\end{array}$ \\
\hline Li et $a l . .^{\mathrm{c}}$ & 633 & & 37.2 & 186 & $\begin{array}{c}\text { Pulsed laser deposition// } \\
\mathrm{Li}_{\mathrm{x}} \mathrm{Ni}_{2-\mathrm{x}} \mathrm{O}(001) / \mathrm{MgO}(001)\end{array}$ & $\begin{array}{c}\text { Polarimetric } \\
\text { Reflection } \\
\text { Sandwich configuration } \\
\text { Interferometric }\end{array}$ \\
\hline This work & 633 & 29.9 & 8.5 & 38.9 & $\begin{array}{l}\text { rf magnetron sputtering// } \\
\mathrm{Pt}(001) / \mathrm{MgO}(001)\end{array}$ & $\begin{array}{c}\text { Reflection } \\
\text { Sandwich configuration }\end{array}$ \\
\hline
\end{tabular}

${ }^{\mathrm{a}}$ Reference 12 .

${ }^{\mathrm{b}}$ Reference 13 .

${ }^{\mathrm{c}}$ Reference 14. 
source of potential error, extensively illustrated in this work, is an inaccuracy of parameter values which may be detected by controlling results consistency, particularly with varying incident angle. Authors quoted in Table II did not proceed to such verifications. We have demonstrated above that, in the absence of such a consistency control, we might have inferred from our measurements erroneous conclusions such as $\left\{r_{13}=30 \mathrm{pm} / \mathrm{V}, r_{33}=5 \times 30=150 \mathrm{pm} / \mathrm{V}\right\}$, that is, values in relative good agreement with previously published results quoted in Table II. In conclusion EO coefficient values previously reported for SBN films in the literature may be suspected of error for various reasons and would need to be confirmed. Only then could EO properties of SBN films be fruitfully discussed with regard to the differences specified in Table II between preparation techniques or selected substrates.

Concerning SBN crystals, very few reports exist in the literature on the separate measurements of $r_{13}$ and $r_{33}$ in SBN crystals. $^{31,32,39,42-44}$ Most of the papers dealing with EO properties of SBN crystals use an assumed value for the ratio $r_{33} / r_{13}$. At $\lambda=633 \mathrm{~nm}$ and for a composition SBN:60, EO coefficients have been determined separately in one paper ${ }^{31}$ which reports: $r_{13}=37 \mathrm{pm} / \mathrm{V}$ and $r_{33}=237 \mathrm{pm} / \mathrm{V}$ from measurements that do not enable specifying signs. Besides the CPE coefficient $d_{33}$ in SBN crystals was reported to be about $95 \mathrm{pm} / \mathrm{V}{ }^{45}$ Compared to their crystalline counterpart, the three coefficients $r_{13}, r_{33}$, and $d_{33}$ of the film appear reduced in similar proportions by a factor about $5 \pm 1$. The magnitude of polarization in our SBN films [see Fig. 5(b)] is also measured about five times lower than that reported for SBN:60 single domain crystals. ${ }^{45}$ These results are consistent with the direct relationship between polarization and EO properties predicted by modeling. ${ }^{46,47}$ Linear EO properties are expected to vanish in unpolarized samples and above coefficients to increase with polarization. The reasons for a lower polarization in our SBN films are under investigation.

Although lower than those of SBN crystals, the application relevant EO coefficients measured in the present work $\left(r_{33}=+38.9 \mathrm{pm} / \mathrm{V}, r_{e f f}=r_{33}-\left(n_{o} / n_{e}\right)^{3} r_{13}=+29.9 \mathrm{pm} / \mathrm{V}\right)$ are larger than those of a crystal of $\mathrm{LN}$ at the same wavelength

$\lambda=633 \mathrm{~nm}$ $\left(r_{33}=+30.9 \mathrm{pm} / \mathrm{V}, r_{e f f}=+20.1 \mathrm{pm} / \mathrm{V}\right),{ }^{48}$ and yield a $V_{\pi} L$ $=0.1 \mathrm{~V} \mathrm{~cm}$ according to Eq. (2). On the other hand the SBN film dielectric permittivity measured in conditions of $\mathrm{EO}$ characterization is about 20 times that of crystalline LN. When referring to the highest value reported for $r_{33}$ in a LN epitaxial thin film ${ }^{3}\left(r_{33} \approx 18 \mathrm{pm} / \mathrm{V}\right)$ and to the present work for SBN, according to Eqs. (2) and (3), it appears that replacing a LN by a SBN thin film should decrease the half-wave voltage-length product by a factor 2.5 but increase the drive power by a factor 3.2. EO performances of SBN and LN thin film paths appear, therefore, comparable. The advantage of SBN path is that it is still far from its crystalline limits. Beside the improvements expected for SBN:60 composition from our understanding of polarization limits, an increase in Sr content $(x>60 \%)$ should induce an enhancement of the film $r_{33}$ coefficient. We have already investigated the SBN:75 composition. Probably because this composition is a limit to the stability of crystalline SBN, multiphase films have been systematically obtained. The composition SBN:65 is under investigation.

\section{CONCLUSION}

Implementation of the linear EO Pockels effect in thin films waveguides is expected to allow drastic reductions in the drive voltage, drive power, and dimensions of light modulation devices. It is also expected to enable the realization of electrically tunable PC devices and thus considerably broaden the potential functionality of these structures. Whereas the understanding of a film electric-field-induced response is of decisive interest for both fundamental and technological reasons, we have brought into relief that its experimental characterization is particularly liable to dramatic errors, caused either by an oversimplified modeling, and/or by a slight inaccuracy in a parameter value.

In this paper, we have presented a method which aims at eliminating systematically the sources of EO characterization unreliability. Based on a Fabry-Perot reflective configuration, it enables characterizing simultaneously the EO, CPE and EA effects in a thin film and provides magnitude and sign of each of the involved coefficients. This characterization method yields a unique solution which accounts for the whole of experimental data: measured reflectivity and electric-field-induced variation in reflectivity, versus incident angle and for both polarizations. The results obtained at $\lambda$ $=633 \mathrm{~nm}$ and room temperature on an epitaxial SBN:60 film are

$$
\begin{aligned}
& r_{13}=+8.5 \pm 1.3 \mathrm{pm} / \mathrm{V}, \\
& d_{33}=\Delta e / \Delta V=+21 \pm 4 \mathrm{pm} / \mathrm{V}, \\
& \Delta k_{o} / \Delta V=(+9.8 \pm 0.6) \times 10^{-6}, \\
& r_{33}=+38.9 \pm 0.5 \mathrm{pm} / \mathrm{V}, \quad \frac{r_{33}}{r_{13}}=+4.5 \pm 0.6 .
\end{aligned}
$$

From our results it appears that CPE and EA effects are significant in the film response, and should not be neglected in any characterization procedure performed on thin films at a frequency below piezoelectric resonance. An elasto-optic part is therefore expected in the EO coefficients, which will collapse at high frequencies above piezoelectric resonance. Our results relative to electroabsorption are to our knowledge the first ones reported concerning SBN. Compared to the corresponding crystalline values, piezoelectric and EO coefficients of the SBN film on one hand, polarization on the other hand, are found to be reduced by approximately the same factor. This correlation is consistent with theoretical predictions. The $r_{33}$ and $r_{\text {eff }}$ EO coefficients of the SBN film explored in the present work are larger than those of a crystal of $\mathrm{LN}$ at the same wavelength $\lambda=633 \mathrm{~nm}$. When taking into account the significant difference in dielectric permittivity between LN and SBN, the two thin film paths appear comparable in terms of EO performance. The advantage of SBN is that our first EO results obtained in this paper are far from the SBN solid solution crystalline limits, leaving place for 
improvement. Increasing the polarization magnitude and modifying the Sr content in the films are the two paths to approach these limits.

${ }^{1}$ M. E. Lines and A. M. Glass, Principles and Applications of Ferroelectrics and Related Materials (Oxford University Press, New York, 1977), p. 592.

${ }^{2}$ P. Rabiei and P. Günter, Appl. Phys. Lett. 85, 4603 (2004).

${ }^{3}$ H. Akazawa and M. Shimada, J. Appl. Phys. 98, 113501 (2005).

${ }^{4}$ P. Tang, D. J. Towner, A. L. Meier, and B. W. Wessels, Appl. Phys. Lett. 85, 4615 (2004).

${ }^{5}$ P. Tang, A. L. Meier, D. J. Towner, and B. W. Wessels, Opt. Lett. 30, 254 (2005).

${ }^{6}$ J. W. Li, F. Duewer, C. Gao, H. Chang, X. D. Xiang, and Y. L. Lu, Appl. Phys. Lett. 76, 769 (2000).

${ }^{7}$ D. Y. Wang, J. Wang, H. L. W. Chan, and C. L. Choy, J. Appl. Phys. 101, 043515 (2007).

${ }^{8}$ K. Sato, M. Kondo, and K. Kurihara, J. Appl. Phys. 102, 054104 (2007).

${ }^{9}$ M. Echizen, T. Fujii, T. Nozaka, T. Nishida, H. Takeda, K. Uchiyama, and T. Shiosaki, Jpn. J. Appl. Phys. 47, 7545 (2008).

${ }^{10}$ M. M. Zhu, Z. H. Du, and J. Ma, J. Appl. Phys. 106, 023113 (2009).

${ }^{11}$ L. D. Zhu, J. Zhao, F. Wang, P. E. Norris, G. D. Fogarty, B. Steiner, P. Lu, B. Kear, S. B. Kang, B. Gallois, M. Sinclair, D. Dimos, and M. CroninGolomb, Appl. Phys. Lett. 67, 1836 (1995).

${ }^{12}$ D. Trivedi, P. Tayebati, and M. Tabat, Appl. Phys. Lett. 68, 3227 (1996).

${ }^{13}$ J. Koo, C. Lee, J. H. Jang, K. No, and B.-S. Bae, Appl. Phys. Lett. 76, $2671(2000)$

${ }^{14}$ X. T. Li, P. Y. Du, H. Ye, C. L. Mak, and K. H. Wong, Appl. Phys. A: Mater. Sci. Process. 92, 397 (2008)

${ }^{15}$ H. Takeda and K. Yoshino, Phys. Rev. E 69, 016605 (2004).

${ }^{16}$ P. T. Lin, Z. Liu, and B. W. Wessels, J. Opt. A, Pure Appl. Opt. 11, 075005 (2009).

${ }^{17}$ R. Kim, J. Zhang, O. Eknoyan, H. F. Taylor, and T. L. Smith, Electron. Lett. 41, 1028 (2005).

${ }^{18}$ M. Roussey, M. P. Bernal, N. Courjal, D. Van Labeke, and F. I. Baida, Appl. Phys. Lett. 89, 241110 (2006).

${ }^{19}$ E. Mishina, A. Zaitsev, N. Ilyin, N. Sherstyuk, A. Sigov, Y. Golovko, V. Muhortov, A. Kolesnikov, Y. Lozovik, M. Yemtsova, and T. Rasing, Appl. Phys. Lett. 91, 041107 (2007).

${ }^{20}$ K. D. Singer, M. G. Kuzyk, W. R. Holland, J. E. Sohn,S. J. Lalama, R. B. Comizzoli, H. E. Katz, and M. L. Schilling, Appl. Phys. Lett. 53, 1800 (1988).

${ }^{21}$ C. A. Eldering, S. T. Kowel, and A. Knoesen, Appl. Opt. 28, 4442 (1989).

${ }^{22}$ R. Meyrueix, J. P. Lecomte, and G. Tapolsky, Nonlinear Opt. 1, 201 (1991).

${ }^{23}$ R. Meyrueix, M. J. Dickens, O. Lemonnier, J. P. Lecomte, and G. Tapolsky, Opt. Commun. 110, 445 (1994).
${ }^{24}$ H. Adachi, T. Kawaguchi, K. Setsune, K. Ohji, and K. Wasa, Appl. Phys. Lett. 42, 867 (1983).

${ }^{25}$ C. C. Teng and H. T. Man, Appl. Phys. Lett. 56, 1734 (1990).

${ }^{26}$ J. S. Schildkraut, Appl. Opt. 29, 2839 (1990).

${ }^{27}$ G. H. Cross, I. R. Girling, I. R. Peterson, and N. A. Cade, Electron. Lett. 22, 1111 (1986).

${ }^{28}$ V. Dentan, Y. Lévy, M. Dumont, P. Robin, and E. Chastaing, Opt. Commun. 69, 379 (1989).

${ }^{29}$ Y. Levy, M. Dumont, E. Chastaing, P. Robin, P. A. Chollet, G. Gadret, and F. Kajzar, Nonlinear Opt. 4, 1 (1993).

${ }^{30}$ B. Pardo, T. Megademini, and J. M. André, Rev. Phys. Appl. 23, 1579 (1988).

${ }^{31}$ H. Y. Zhang, X. H. He, Y. H. Shih, and S. H. Tang, Opt. Commun. 86, 509 (1991).

${ }^{32}$ P. V. Lenzo, E. G. Spencer, and A. A. Ballman, Appl. Phys. Lett. 11, 23 (1967).

${ }^{33}$ M. Cuniot-Ponsard, J. M. Desvignes, B. Ea-Kim, and E. Leroy, J. Appl. Phys. 93, 1718 (2003).

${ }^{34}$ M. Cuniot-Ponsard, J. M. Desvignes, and A. Bellemain, J. Mater. Sci. 41, 5302 (2006).

${ }^{35}$ N. Boulay, M. Cuniot-Ponsard, J. M. Desvignes, and A. Bellemain, Ferroelectrics 353, 10 (2007).

${ }^{36}$ F. Bridou and B. Pardo, J. Opt. 21, 183 (1990).

${ }^{37}$ E. L. Venturini, E. G. Spencer, P. V. Lenzo, and A. A. Ballman, J. Appl. Phys. 39, 343 (1968).

${ }^{38}$ E. L. Venturini, E. G. Spencer, and A. A. Ballman, J. Appl. Phys. 40, 1622 (1969).

${ }^{39}$ M. Abarkan, J. P. Salvestrini, M. D. Fontana, and M. Cuniot-Ponsard, Appl. Phys. B: Lasers Opt. 91, 489 (2008).

${ }^{40}$ Y. Xu, J. Chen, R. Xu, and J. D. Mackenzie, Phys. Rev. B 44, 35 (1991).

${ }^{41}$ A. D. Li, J. Tan, W. C. Liu, C. L. Mak, K. H. Wong, H. Ye, D. Wu, and N. B. Ming, Ferroelectrics 332, 159 (2006).

${ }^{42}$ S. Ducharme, J. Feinberg, and R. R. Neurgaonkar, IEEE J. Quantum Electron. 23, 2116 (1987).

${ }^{43}$ K. Onuki, N. Uchida, and T. Saku, J. Opt. Soc. Am. 62, 1030 (1972).

${ }^{44}$ M. Goulkov, T. Granzow, U. Dörfler, Th. Woike, M. Imlau, R. Pankrath, and W. Kleeman, Opt. Commun. 218, 173 (2003).

${ }^{45}$ A. M. Prokhorov and S. Yu, Kuz'minov, Ferroelectric Crystals for Laser Radiation Control (IOP, Bristol, 1990), p. 149.

${ }^{46}$ M. E. Lines and A. M. Glass, Principles and Applications of Ferroelectrics and Related Materials (Oxford University Press, New York, 1977), p. 477.

${ }^{47}$ J. R. Oliver, R. R. Neurgaonkar, and L. E. Cross, J. Appl. Phys. 64, 37 (1988).

${ }^{48}$ David N. Nikogosyan, Non Linear Optical Crystals: A Complete Survey (Springer, New York, 2005), p. 35.

${ }^{49}$ Handbook of Optical Constants, edited by E. D. Palik (Academic, Orlando, 1985). 